mit ihren Erkenntnissen gehört $\mathrm{zu}$ werden, ohne ihre wissenschaftliche Distanz auf dem »Altar« der Mediengesellschaft zu opfern.

- Grundvoraussetzung einer wissenschaftlich fundierten Mitgestaltung ist eine starke Kriminologie an den Universitäten, Fachhochschulen und Ausbildungseinrichtungen möglichst aller Berufsfelder, die mit den »Kriminalität« genannten Phänomenen in Berührung kommen. Einhellig warnten verschiedene Redner während des Kriminologietages davor, die Kriminologie im Zuge von Sparoder Umstrukturierungsmaßnahmen in den Curricula oder Lehrstuhlwidmungen zurückzudrängen oder gar verschwinden $\mathrm{zu}$ lassen. Insbesondere die strafjuristische Ausbildung könne ohne ihre kriminologischen und rechtssoziologischen Bezüge nicht sinnvoll betrieben werden. Eine starke Kriminologie ist wiederum angewiesen auf qualifizierte Forscher unterschiedlicher Disziplinen, die diese Wissenschaft auch tatsächlich be- und vorantreiben. Dafür reicht es nicht aus, sich mit der drohenden Situation zufrieden zu geben, dass - wie es ein Tagungsbesucher formulierte - »jeder, der ein Kriminologiebuch vorlesen kann, auch Kriminologie macht, nur weil es eben zufällig auf dem Lehrplan steht«. Durch die engagiert geführten Diskussionen und die deutliche Annäherung zwischen Mitgliedern der großen Fachgesellschaften hat der erste Kriminologietag zu einer Stärkung dieser Disziplin bereits beigetragen. Eine regelmäßige Wiederholung derartiger Tagungen ist überaus wünschenswert und zu erwarten.

Dr. jur. Michael Jasch ist wissenschaftlicher Mitarbeiter und Lehrbeauftragter am Institut für Kriminalwissenschaften der Johann Wolfgang Goethe-Universität Frankfurt a.M., FB Rechtswissenschaft, sowie Geschäftsführer der Gesellschaft für interdisziplinäre wissenschaftliche Kriminologie (GiwK), Jasch@jur.uni-frankfurt.de

\title{
Strafverteidigertag: Feindstrafrecht und Willensfreiheit
}

Christine Morgenstern

G roße Fragen auf dem 29. Strafverteidigertag: Brauchen wir ein Parallel-, ein Feindstrafrecht? Oder brauchen wir vielleicht gar keines mehr? Die Veranstalter hatten noch etwas bescheidener, aber nicht weniger grundsätzlich gefragt: »Wen schützt das Strafrecht?« Die 500 Teilnehmer beschäftigten sich vom 4 . - 6. März 2005 in Aachen unter diesem Generalthema mit dem Spannungsverhältnis zwischen Sicherheit und Freiheit. In sechs Arbeitsgruppen ging es um immer längere Strafen und die Sicherungsverwahrung; um gesperrte Zeugen und gesperrte Akten; neue Erkenntnisse zu Willensfreiheit; um das Folterverbot; um europäische Entwicklungen und um die Strafprozessreform.

Konkretisiert wurde das Thema am Freitagabend im engagierten Eröffnungsvortrag von Prof. Dr. Bernhard Haffke, Passau, "Vom Rechtssaat zum Sicherheitsstaat? «Ihm ist eine Erweiterung der klassisch-abwehrrechtlichen Sicht wichtig, die sich auf den Schutz vor Eingriffen des Staates in die Freiheitsrechte des Bürgers konzentriert. Dabei will er sich allerdings nicht dem »Mainstream zeitgenössischen Sicherheitsdenkens « ${ }^{1}$ anschließen, sondern die in der Rechsprechung des Bundesverfassungsgerichts und in der Literatur erarbeiteten Figuren, etwa der "grundrechtlichen Schutzpflichten « oder des "Grundrechts auf Sicherheit«, reflektieren, die ihrerseits auf Wandlungen des Staates hin zu einem »Risikobeherrschungsstaat « reagieren. Mit anderen Worten: Das Strafrecht, obgleich als Reaktion auf begangenes Unrecht konzipiert, »entrinnt nicht der Herrschaft des prospektiven, des präventiven Sicherheitsparadigmas«, es bedarf also einer systemischen Gesamtschau. Er weist in diesem Zusammenhang auf den Wandel des Menschenbildes hin, der nicht erst seit Herbst 2001 - die moderne Gesetzgebung beherrscht: Es ist nun offenbar von Misstrauen geprägt; im Gefahrenvorfeld wird dem Bürger nicht mehr Redlichkeit unterstellt, sondern das Gegenteil ist der Fall, und der Unredliche ist "potenziell gefährlich und muss deshalb beobachtet werden.« Er warnt dann eindringlich davor, das Wuchern von Gefährdungsbekämpfungs- oder »Risikosteuerungsgesetzen«, die alles Heil im Vorrang der Prävention vor der Repression sehen, zu akzeptieren. Er nennt dabei als Beispiele das Terrorund das Geldwäschebekämpfungsgesetz oder das jüngst in Kraft getretene Gesetz zur Förderung der Steuerehrlichkeit; aber auch jene Gesetze, die die Schwelle für Gefahrermittlungseingriffe deutlich herabsetzen, wie zum Beispiel das G10-Gesetz; zeigt auch offen sein Misstrauen gegenüber allem, was das Strafrecht "therapeutisieren " will. Es ist Haffke ein Anliegen, darauf hinzuweisen, dass dieser weitgehenden Sozialkontrolle unter dem Etikett der Prävention nicht dadurch ausgewichen werden kann, dass man solche Aspekte aus dem Strafrecht und Strafprozessrecht sauber heraushält und in ein $\mathrm{Ge}$ fahrenabwehrrecht, d. h. in das Polizeirecht verlagert.

Der Samstag war den sechs Arbeitsgruppen gewidmet, die inhaltlich durch das gut zusammengestellte Materialheft vorbereitet werden konnten. Die Ergebnisse sind inzwischen im Internet unter www.strafverteidigervereinigungen.de abrufbar. Die Arbeitsgruppe 1, die sich mit (lebens-)langen Freiheitsstrafen und der Sicherungsverwahrung beschäftigt hatte, wiederholt alte, aber eben leider nicht obsolete Forderungen unter anderem nach Abschaffung jeglicher Form der Sicherungsverwahrung und verlangt eine Standardisierung und Verbesserung von Prognosegutachten. Die Arbeitsgruppe 2 "Sicherheitsstaat versus Fair Trial/Gesperrte Zeugen und gesperrte Akten« erhebt vor allem die Forderung, dass die staatliche Zurückhaltung von Beweismitten (durch Sperrerklärungen etc.) zumindest in vielen Fällen zu einem Verfahrenshindernis führen muss. Arbeitgruppe 4 nahm sich des Folterverbots bzw. vorgelagerter Maßnahmen an. Hier ist neben der Absage an jegliche Relativierung des Folterverbots die Forderung wichtig, das Zusatzprotokoll zur UN-Antifolterkonvention zügig $\mathrm{zu}$ ratifizieren und umzusetzen. Außerdem müsse ein nationales Gremium zur Kontrolle insbesondere in den Haftanstalten eingesetzt werden. Die Arbeitsgruppe 5 »Europäischer Sicherheitsstaat - Europäischer Polizeistaat? « kritisiert in ihrem Ergebnispapier sehr grundsätzlich die rein sicherheitsorientierte, »bürokratische und utilitaristische« Brüsseler Rechtssetzung in Straf- und Strafverfahrensrecht, die häufig eine Angleichung auf den niedrigsten Rechtsstandard mit sich bringe und konkretisiert das am Beispiel des Europäischen Haftbefehls. Die Arbeitsgruppe befürwortet weitere Vernetzungen und auch die Einrichtung einer "Selbsthilfeorganisation" der Strafverteidiger auf europäischer Ebene, wie etwa einen »Eurodefensor «. Die Arbeitsgruppe 6 schließlich befasste sich mit der Strafprozessreform. Sie bedauert, dass die Vorschläge des Diskussionsentwurfs für eine Reform des Strafverfahrens vom Februar 2004 durch die Justizminister der Länder nicht nur nicht vorangebracht wurden, sondern eher eine "Gegenreform « zu verzeichnen sei. Sie erneuert bzw. erweitert unter anderem die Forderungen nach Ausdehnung der notwendigen Verteidigung für alle Verfahren vor dem Schöffengericht und nach technischen Aufzeichnungen aller Vernehmungen im Ermittlungsverfahren sowie der gesamten Hauptverhandlung.

Die Verfasserin dieses Beitrags nahm an der Arbeitsgruppe $3 \mathrm{mit}$ dem kryptischen Titel »Tun wir, was wir wollen oder wollen wir, was wir tun?« teil. Hier ging es um neue Erkenntnisse der Hirnforschung zur Willensfreiheit und den Diskurs mit Philosophen und - zögernd - auch Juristen, für die diese Frage nach der Existenz oder der bloßen Illusion der Willensfreiheit zentrale Bedeu- 
tung insbesondere im Hinblick auf das Schuldprinzip des Strafrechts hat. Die Veranstalter hatten gut geplant und viel Zeit für Diskussionen gelassen, die von den Teilnehmerinnen und Teilnehmern der sehr gut besuchten Arbeitsgruppe auch lebhaft in Anspruch genommen wurde. Man merkte deutlich, dass trotz des von den klassischen Anliegen eines Strafverteidigertages doch relativ weit entfernten Themas mit interdisziplinärem Ansatz bei den Zuhörern entsprechender Diskussionsbedarf vorhanden war: Trieb die einen die Sorge, die Hirnforscher könnten durch ihre neuesten Forschungsergebnisse das Postulat der Willensfreiheit endgültig als Fiktion entlarven und so dem Strafrecht seine Grundlage entziehen; war es bei den anderen die Hoffnung, durch derartige Ergebnisse bekämen sie nun endlich die Strategie an die Hand, die Mandanten als für ihre konkrete Tat nicht selbst verantwortlich verteidigen $\mathrm{zu}$ können.

Zunächst referierte Prof. Dr. Benedikt Grothe, von der Abteilung Biologie II der LMU München. Er führte kurz in die Geschichte der Hirnforschung ein und wies darauf hin, dass es für den hier besprochenen Bereich gar nicht so viele neue Erkenntnisse, sondern »lediglich" sensationelle neue Mess- und Darstellungsmethoden gebe, die allerdings für andere Bereiche in der Tat dramatische Zugewinne brächten. Er führte hier als Beispiel die Möglichkeiten an, Nervenfunktionen durch mikroelektronische Systeme zu ersetzen, mit denen man Prothesen steuern kann (so etwa elektronische Hörprothesen, die direkt am Hirnstamm eingesetzt werden). Er besprach die in der Diskussion allgegenwärtigen Experimente, die Benjamin Libet bereits 1983 in den USA durchführte und die nachwiesen, dass im sog. prämotorischen Cortex bereits deutlich vor dem Zeitpunkt, in dem die Probanden angaben, sich zu einer bestimmten, später ausgeführten Körperbewegung entschieden zu haben, ein messbares Bereitschaftspotenzial entstanden war. Nach Auffassung vieler Wissenschaftler, darunter auch Grothe selbst, sind diese (wie auch später durchgeführte ähnliche) Experimente aber nicht geeignet, eine Entscheidungssituation mit Relevanz für die Frage der freien Willensbildung modellhaft nachzubilden, da die Probanden instruiert waren, eine bestimmte Bewegung auszuführen. Diese Handlung im Libet-Experiment ist ethisch und emotional irrelevant, so dass über entsprechend relevante Handlungen, die etwa für das Strafrecht bedeutsam wären, keine Aussage getroffen werden kann - möglicherweise werden hier ganz andere Hirnmechanismen aktiviert. Im Gegensatz zu namhaften Hirnforschern wie etwa Singer, Prinz oder Roth, ${ }^{2}$ schließt sich deshalb Grothe der Auffassung derjenigen an, die festhalten, dass die unvorstellbare Komplexität des Gehirns Extrapolationen, die aus Befunden von reduktionistisch gestalteten Experimenten abgleitet werden, keine Schlüsse zulassen auf komplexe natürliche Situationen und die Hirnmechanismen, die diese bewältigen. Es ist damit allerdings nicht gesagt, das betont auch Grothe, dass Aussagen hierüber nicht irgendwann getroffen werden können. Käme man dann tatsächlich dazu, dass unser Gehirn stets schon für uns entschieden hat, bevor wir es bewusst tun, ist noch die Frage, ob sich das Menschenbild negativ verändern müsste, so Grothe in der Diskussion: Auch dann würde die beschriebene Komplexität, die vom Individuum einen so hohen Grad an Selbstorganisation verlangt, wohl ausreichend Respekt abnötigen. Beeindruckend waren in diesem $\mathrm{Zu}$ sammenhang im übrigen die Darstellungen zu Dynamik und Adaptivität neuronaler Systeme, die zumindest aus der Sicht des Neurobiologen viel Hoffnung machen was die Lern- und Anpassungsfähigkeit unseres Gehirns - und damit die Möglichkeit, auch Verhaltensänderungen bei Straftätern herbeizuführen - angeht.

Um den nötigen interdisziplinären Ansatz zu komplettieren (einzig die Theologie war nicht vertreten, das wäre dann für einen Strafverteidigertag wohl doch zu viel gewesen), referierte im Anschluss Dr. Bettina Walde vom Philosophischen Seminar der Johannes-GutenbergUniversität Mainz. Sie wies darauf hin, dass die durch die diskutierten Forschungsergebnisse (oder deren Interpretation) aufgeworfenen Fragen nach der Willensfreiheit letztlich Varianten der bekannten Diskussion um Determinismus und Indeterminismus sind. Hier stehen sich zwei miteinander unvereinbare Forderungen gegenüber: Einerseits beanspruchen wir als handelnde
Individuen, dass zumindest einige unserer Handlungen frei sind im Sinne von snicht determiniert . Andererseits scheint der Determinismus der Physik unumgänglich. Während kompatibilistische Ansätze versuchen, den Determinismus der Physik mit der Freiheit verträglich zu machen (etwa durch ein »Herunterschrauben « des involvierten Freiheitsbegriffs), gehen inkompatibilistische Ansätze davon aus, dass Determinismus und Freiheit in keinem Fall miteinander vereinbar sind. Solche Argumentationen setzen sehr häufig einen starken Freiheitsbegriff voraus, wonach der Wille als »unbewegter Beweger « verstanden, neue Kausalketten in Gang setzen kann. Schwächt man hingegen den Freiheitsbegriff dahingehend $a b$, dass er als relative Autonomie des handelnden Individuums $\mathrm{zu}$ verstehen ist, das in subjektiver Entscheidungsfreiheit handelt (und das man dementsprechend hierfür auch zur Verantwortung ziehen kann), kann man das (vermeintliche) Dilemma auflösen.

Ein Referat des Hamburger Professors für Strafrecht und Rechtsphilosophie Reinhard Merkel bildete den Abschluss der Arbeitsgruppensitzung. Für ihn ist die Debatte um die Willensfreiheit keineswegs ausgestanden, in der Abschlussdiskussion mit ihm war auch am deutlichsten das schwierige Ringen mit dem Thema zu spüren. Er machte nochmals deutlich, welche Konsequenzen es hätte, wenn dem Strafrecht das Prinzip der Verantwortungszuschreibung, das auf der Idee der Willensfreiheit basiert, genommen würde: Eine Bestrafung hätte dann nur noch eine gleichsam mechanische Funktion der Vergeltung, nicht aber die, eine gebrochene Norm wiederherzustellen oder den Täter zu resozialisieren. Da dies wohl nicht zu akzeptieren wäre, müsste das Strafrecht abgelöst werden durch ein Gefahrenabwehrrecht, das gefährliche Täter verwahrt bzw. ggf. zu therapieren versucht. Welche enormen Gefahren - zum Beispiel die völlige Abhängigkeit von prognostischen Gutachten - ein solches zum Beispiel für den Strafvollzug mit sich bringen würde, mag man sich gar nicht ausdenken.
Den Abschluss der Tagung bildete am Sonntagvormittag die Schlussdiskussion »Vom Bürger- und Feindstrafrecht? «. Auf dem Podium saßen Prof. Günter Jakobs aus Bonn, Prof. Rainer Hamm aus Frankfurt, Prof. Bernhard Haffke und der Bundesanwalt Rainer Griesbaum. Erwartungsgemäß schossen sich die Teilnehmer der Diskussion schnell und heftig auf Jakobs ein, der damit ausreichend Gelegenheit bekam, unverändert und beharrlich sein Konzept des Feindstrafrechts zu vertreten (im Materialheft war der auch online erschienene Beitrag »Bürgerstrafrecht und Feindstrafrecht $\aleph^{3}$ enthalten). Bei seinem Ausgangspunkt, der mit der richtigen Beobachtung, dass bereits jetzt das Strafrecht von aus rechtsstaatlicher Sicht als Fehlentwicklung zu charakterisierenden "feindstrafrechtlichen Einsprengseln « durchsetzt ist - er nennt dabei z. B. die Sicherungsverwahrung oder die Terrorismusgesetzgebung als Fremdkörper - , ist ihm Beifall noch sicher. Immer deutlicher wird, dass er diese Entwicklung aber legitim findet, lediglich dazu auffordert, methodisch sauber zu arbeiten: Bürgerstrafrecht und Feindstrafrecht sollen sauber voneinander getrennt werden, um nicht demjenigen, der zwar das Gesetz bricht, grundsätzlich aber noch als sein Adressat in Frage kommt (»ansprechbar ist«) mit der gleichen Härte zu treffen, die gegenüber Feinden angebracht sei. Ein Feind ist, so definiert es Jakobs, derjenige, der keine »kognitive Garantie" mehr dafür gibt, dass er sich als Person verhalten will, $d . h$. wenigstens potenziell auf das Recht ansprechen wird. Als Beispiel hierfür nennt er immer wieder den »Terroristen «. Hatte man bei der Lektüre seiner Beiträge 4 immer wieder Zweifel, ob er wirklich das meinen kann, was sich aufdrängt, wurde es in der Diskussion von ihm selbst bestätigt: Ja, wenn Guantanamo zur Bekämpfung solcher Gefahren für den Staat nötig sei, was er allerdings nicht wisse, dann sei es über das Modell des Feindstrafrechts zu rechtfertigen. $\mathrm{Zu}$ beurteilen, ob und für wen ein solches Vorgehen nötig sei, sei nicht seine Sache. Spätestens hier gaben die meisten die Diskussion auf und wandten gar nicht mehr ein, dass er nebenbei gerade auch noch das Kriegsvölkerrecht abgeschafft hatte. Immerhin war er noch zu der Aus- 
sage $\mathrm{zu}$ bewegen, dass auch nach seiner Auffassung nicht per se alles erlaubt sei, sondern dass der Staat quasi aus Selbstachtung auch den Feind menschenwürdig behandeln müsse. Hierzu zwingt ihn aber nach Jakobs' Auffassung - dann nicht mehr das Straf- oder Strafprozessrecht.

Die Diskussion war durch diesen Beitrag sehr stark geprägt, so dass andere wichtige Aspekte, die auch in den Arbeitsgruppen bedeutsam waren, nur noch am Rande Aufmerksamkeit fanden. Sowohl Griesbaum als auch Haffke wiesen aber an verschiedenen Stellen darauf hin, dass einer Entwicklung, die lediglich
Straf- und Strafprozessrecht "rein « hält von Gefahrenabwehr und diese dem Polizeirecht überlässt, mit Sorge $\mathrm{zu}$ betrachten ist. Wer tatsächlich fordert, für die »gefährlichen Normalen « oder andere problematische Gruppen spezielle Gesetze zu schaffen - dies klang in den Diskussionen teilweise an -, wird seine Mandanten demnächst eben nicht mehr in U-Haft oder im Strafvollzug aufsuchen, sondern in Polizeihaft. Es ist dann aber zu befürchten, dass die Rechtsgarantien schwächer sind als die des Straf- und Strafprozessrechts. Insofern muss es darum gehen, staatliche Eingriffe insgesamt auf das notwendige Minimum zu beschränken, die systemische Gesamtschau, die dazu notwendig und die mit Haffke zu fordern ist, ist interdisziplinär zu leisten.

Dr. Christine Morgenstern ist wissenschaftliche Mitarbeiterin am Lehrstuhl für Kriminologie der Ernst-Moritz-Arndt-Universität Greifswald

\section{Fußnoten:}

1 Der Vortrag ist als pdf-Datei abrufbar unter www.strafverteidigervereinigungen.de.

2 Die Diskussionen fanden nicht nur in der Fachliteratur, sondern auch in den Feuilletons der FAZ, der ZEIT, der Süddeutschen Zeitung oder der Frankfurter Rundschau statt. Einen guten Überblick gewinnt man auch, wenn man die
Debatte in der Deutschen Zeitschrift für Philosophie (Jg. 52, 2004), an der u. a. Wolf Singer, Gerhard Roth und Jürgen Habermas beteiligt waren, verfolgt.

3 Abrufbar unter http://www.hrrstrafrecht.de/hrr/archiv/04-03.

4 Jakobs a.a.o.; außerdem in: Eser/ Hassemer/Burkhardt (Hrsg.): Die Deutsche Strafrechtswissenschaft vor der Jahrtausendwende. Rückbesinnung und Ausblick. München 2000, S. 47 ff. Die kritischen Auseinandersetzungen sind inzwischen zahlreich; auch sie haben teilweise den Weg in die überregionalen Tageszeitungen gefunden, vgl. z. B. Prantl in der Suddeutschen Zeitung vom 5./6.3.2005, S. 17; Lüderssen in der FR vom 28.1.2002; auch Düx ZRP 2003, S. $189 \mathrm{ff}$.

\section{Neuregelungen des Menschenhandels - bewusste Regelung oder Anpassung an europäische Vorgaben in normativer Unfreiheit?}

Monika Frommel

In der letzten Aprilwoche wurde vor dem Bundesverfassungsgericht über den Europäischen Haftbefehl verhandelt. Der Berichterstatter Udo Di Fabio überraschte durch eine geradezu frappierende Offenheit. Der deutsche Gesetzgeber sei keinesfalls gezwungen - sozusagen in normativer Unfreiheit - alles zu ratifizieren und umzusetzen, was in einer europäischen Richtlinie als Vorgabe beschlossen worden sei. Der deutsche Gesetzgeber sei im Bereich des Strafund Strafverfahrensrechts nicht so stark an europäische Vorgaben gebunden wie im europäischen Zivilrecht, sondern stimme der Sache nach lediglich einem völkerrechtlichen Vertrag zu, da der Zweck der EU eine Wirtschaftseinheit sei und gerade nicht ein mit Zwangsgewalt ausgestatteter Bundesstaat. Wir werden sehen, wie diese Debatte weiter gehen wird. Jedenfalls wirft sie ein grelles Licht auf die ungute Übung seit einiger Zeit, europäische Rahmenbeschlüsse »durchzuwinken«. Bei den auf der nächsten Seite abgedruckten Neuregelungen des Menschenhandels ist der Widerspruch zum deutschen ProstG 2002, das schließlich eine Legalisierung der Prostitution mit sich gebracht hat, offenkundig; denn wie kann die Vermittlung erwachsener Menschen in die Prostitution strafbar sein, wenn sie als Vertragspartner Rechte und Pflichten übernehmen können (bei den unter 21-Jährigen verlangt § 232 nicht mehr wie die Qualifizierung in $\S 180$ b Abs. 2 Nr. 2 StGB alt die Kenntnis einer Zwangslage). Friedrich Christian Schroeder hat in der NJW $(20 / 2005,1393)$ ausführlich die Kompliziertheit des europäischen Rahmenbeschlusses und deren Umsetzung beschrieben. Offenbar fällt es schwer, beim Thema Frauen- und Kinderhandel einen klaren Kopf zu behalten und die Be- troffenen nicht nur als Opfer (was sie sein können), sondern auch als Akteure zu begreifen, welche auf riskante Weise versuchen, aus einer hoffnungslosen in eine ausbaufähige wirtschaftliche Lage zu gelangen. Auch das Schweizer Recht (vgl. insoweit SZK 1/2005) folgt dem schlechten europäischen Vorbild (allerdings trieft die frühere Regelung vor Moralisierungen) und auch das österreichische Recht fällt durch Überregulierung im Strafrecht auf, allerdings regelt es den gesamten Komplex des Menschenhandels im Sexualstrafrecht, was den Verdacht aufkommen lässt, man möchte nun doch wieder zu Regulierungen von »Unzucht « zurückkehren. Es war zu befürchten, dass die Kontrolle grauer und schwarzer Märkte zu unklarem Strafrecht führen würde, aber etwas mehr Konsequenz hätte ich mir von einer europäischen und natio- nalen Gleichstellungspolitik erwartet. So kann ich nur einen Schulterschluss mit Konservativen feststellen, welche nicht an besseren Verhältnissen für Prostituierte interessiert sind, sondern der Straflosigkeit der bloßen Förderung der Prostitution nachtrauern und sich nun diese polizeilichen verlorenen Befugnisse über das Ausländerrecht, flankiert von einem neuen Strafrecht, wieder verschaffen. Damit sind die "Lichtblicke in der Schattenwelt « (so die SZ vom 23.05.2005, Die Seite Drei) wieder verdunkelt; und zwar nicht nur deswegen, weil nach der Wahl in NRW Netzwerke wie in Dortmund ins Hintertreffen geraten und eine tiefschwarze Politik wie die der Bayerischen Polizeibehörden näher rückt, welche bekanntlich besonders gerne die rotgrüne Rathausmehrheit mit ihrer CSU-Politik im Bereich der Landeszuständigkeiten ärgern. 